\title{
HERMITE-HADAMARD TYPE INEQUALITIES WITH APPLICATIONS
}

\author{
M. U. AWAN, M. A. NOOR, F. SAFDAR, A. ISLAM, M. V. MIHAI, AND K. I. NOOR
}

Received 30 January, 2019

\begin{abstract}
The main objective of this article is to obtain some new estimates related to HermiteHadamard-like integral inequalities essentially using the class of $h$-convex functions. For this we first derive a new integral identity which will serve as an auxiliary result for obtaining the main results of the article. Several new and known special cases are also discussed. In the last some applications to means of real numbers are also discussed in detail which elaborates the significance of the obtained results.
\end{abstract}

2010 Mathematics Subject Classification: 26D15; 26A51; 26E60

Keywords: convex, $h$-convex, Hermite-Hadamard, inequalities, means

\section{INTRODUCTION AND PRELIMINARIES}

Convexity due to its numerous applications has attracted considerable number of researchers. As a result several new generalizations of classical convexity have been obtained in the literature. For example, see [2, 3, 8, 9, 11, 12]. Among these new generalizations $h$-convexity which is due to Varosanec [14] is one of the most charming generalization of classical convexity. It not only unifies the classical convexity but also some of other its generalizations, such as it generalizes Breckner type of $s$-convexity [1], Godunova-Levin type of $s$-convexity [4], $P$ type of functions [7], $Q$ type of functions [10] and $t g s$-convex functions [13].

The class of $h$-convex functions reads as:

Definition 1. Let $h:(0,1) \rightarrow \mathbb{R}$ be a real function. We say that $f: I \rightarrow \mathbb{R}$ is an $h$-convex function, if

$$
f(t x+(1-t) y) \leq h(t) f(x)+h(1-t) f(y), \forall x, y \in I, t \in(0,1) .
$$

Now one can easily observe that for different suitable choices of the function $h($.$) ,$ we have different classes of convex functions. For details, see [14]. Another fascinating aspect of theory of convexity is its close link with theory of inequalities. Many inequalities are direct consequences of the applications of classical convexity. A result in this regard, which is due to Hermite and Hadamard and is commonly known as Hermite-Hadamard's integral inequality. It reads as: 
Theorem 1. Let $f: I=[a, b] \subset \mathbb{R} \rightarrow \mathbb{R}$ be a convex function. If $f \in L[a, b]$, then

$$
f\left(\frac{a+b}{2}\right) \leq \frac{1}{b-a} \int_{a}^{b} f(x) \mathrm{d} x \leq \frac{f(a)+f(b)}{2} .
$$

This result through its simplicity yet having a number applications has attracted several researchers from all over the world. Consequently many new generalizations of this result have been derived in the literature. For more information, see [5, 6, 11, $12,15]$.

The main objective of this article is obtain some new refinements of HermiteHadamard's integral inequality. For this we derive a new integral identity for differentiable functions. This identity will serve as an auxiliary result for obtaining main results of the article. We also discuss several new special cases in detail. In the last we present applications of some of the proposed results to means of real numbers.

\section{Auxiliary Result}

In this section, we derive new integral identity which will play significant role in obtaining main results of the article. Before we move further, for simplicity, we let

$$
\Omega_{1}=\frac{n}{n+1} a+\frac{1}{n+1} b \text { and } \Omega_{2}=\frac{1}{n+1} a+\frac{n}{n+1} b .
$$

Lemma 1. Let $f: I \rightarrow \mathbb{R}$ be differentiable function on $I^{o}, a, b \in I$ with $a<b$. If $f^{\prime} \in L_{1}[a, b], 0 \leq \lambda, \mu \leq 1$ and $n \in \mathbb{N}^{*}$, then we have

$\phi(n ; \lambda, \mu ; a, b)-\frac{1}{b-a}\left[\int_{a}^{\Omega_{1}} f(x) \mathrm{d} x+\int_{\Omega_{2}}^{b} f(x) \mathrm{d} x\right]$
$=\frac{b-a}{(n+1)^{2}} \times \int_{0}^{1}\left[(1-\lambda-t) f^{\prime}\left(\frac{n+t}{n+1} a+\frac{1-t}{n+1} b\right)+(\mu-t) f^{\prime}\left(\frac{t}{n+1} a+\frac{n+1-t}{n+1} b\right)\right] \mathrm{d} t$,

where

$$
\begin{aligned}
& \phi(n ; \lambda, \mu ; a, b) \\
& =\frac{1}{n+1}\left[\lambda f(a)+(1-\lambda) f\left(\frac{n}{n+1} a+\frac{1}{n+1} b\right)+(1-\mu) f\left(\frac{1}{n+1} a+\frac{n}{n+1} b\right)+\mu f(b)\right] .
\end{aligned}
$$

Proof. Take

$$
\begin{aligned}
I & =\frac{b-a}{(n+1)^{2}} \int_{0}^{1}\left[(1-\lambda-t) f^{\prime}\left(\frac{n+t}{n+1} a+\frac{1-t}{n+1} b\right)+(\mu-t) f^{\prime}\left(\frac{t}{n+1} a+\frac{n+1-t}{n+1} b\right)\right] \mathrm{d} t \\
& =\frac{b-a}{(n+1)^{2}}\left(I_{1}+I_{2}\right) .
\end{aligned}
$$

Now

$$
I_{1}=\int_{0}^{1}(1-\lambda-t) f^{\prime}\left(\frac{n+t}{n+1} a+\frac{1-t}{n+1} b\right) \mathrm{d} t
$$




$$
=\frac{n+1}{b-a}\left[\lambda f(a)+(1-\lambda) f\left(\frac{n}{n+1} a+\frac{1}{n+1} b\right)-\int_{0}^{1} f\left(\frac{n+t}{n+1} a+\frac{1-t}{n+1} b\right) \mathrm{d} t\right] .
$$

Putting $x=\frac{n+t}{n+1} a+\frac{1-t}{n+1} b$ in the above equality, we have

$$
I_{1}=\frac{n+1}{b-a}\left[\lambda f(a)+(1-\lambda) f\left(\frac{n}{n+1} a+\frac{1}{n+1} b\right)-\frac{n+1}{b-a} \int_{a}^{\Omega_{1}} f(x) \mathrm{d} x\right],
$$

and

$$
\begin{aligned}
I_{2} & =\int_{0}^{1}(\mu-t) f^{\prime}\left(\frac{t}{n+1} a+\frac{n+1-t}{n+1} b\right) \mathrm{d} t \\
& =\frac{n+1}{b-a}\left[(1-\mu) f\left(\frac{1}{n+1} a+\frac{n}{n+1} b\right)+\mu f(b)-\int_{0}^{1} f\left(\frac{t}{n+1} a+\frac{n+1-t}{n+1} b\right)\right] \mathrm{d} t .
\end{aligned}
$$

Using $x=\frac{t}{n+1} a+\frac{n+1-t}{n+1} b$ in above equality, we have

$$
I_{2}=\frac{n+1}{b-a}\left[(1-\mu) f\left(\frac{1}{n+1} a+\frac{n}{n+1} b\right)+\mu f(b)-\frac{n+1}{b-a} \int_{\Omega_{2}}^{b} f(x) \mathrm{d} x\right] .
$$

Utilizing the values of $I_{1}$ and $I_{2}$ from (2.2) and (2.3) in (2.1), we have

$$
\begin{aligned}
I= & \frac{b-a}{(n+1)^{2}} \frac{n+1}{b-a}\left[\lambda f(a)+(1-\lambda) f\left(\frac{n}{n+1} a+\frac{1}{n+1} b\right)\right. \\
& \left.-\frac{n+1}{b-a} \int_{a}^{\Omega_{1}} f(x) \mathrm{d} x+(1-\mu) f\left(\frac{1}{n+1} a+\frac{n}{n+1} b\right)+\mu f(b)-\frac{n+1}{b-a} \int_{\Omega_{2}}^{b} f(x) \mathrm{d} x\right] \\
= & \frac{1}{n+1}\left[\lambda f(a)+(1-\lambda) f\left(\frac{n}{n+1} a+\frac{1}{n+1} b\right)+(1-\mu) f\left(\frac{1}{n+1} a+\frac{n}{n+1} b\right)+\mu f(b)\right] \\
& -\frac{1}{b-a}\left[\int_{a}^{\Omega_{1}} f(x) \mathrm{d} x+\int_{\frac{1}{n+1} a+\frac{n}{n+1} b}^{b} f(x) \mathrm{d} x\right] .
\end{aligned}
$$

This implies

$$
\begin{aligned}
& \phi(n ; \lambda, \mu ; a, b)-\frac{1}{b-a}\left[\int_{a}^{\Omega_{1}} f(x) \mathrm{d} x+\int_{\Omega_{2}}^{b} f(x) \mathrm{d} x\right] \\
& =\frac{b-a}{(n+1)^{2}} \int_{0}^{1}\left[(1-\lambda-t) f^{\prime}\left(\frac{n+t}{n+1} a+\frac{1-t}{n+1} b\right)+(\mu-t) f^{\prime}\left(\frac{t}{n+1} a+\frac{n+1-t}{n+1} b\right)\right] \mathrm{d} t,
\end{aligned}
$$

where

$$
\begin{aligned}
& \phi(n ; \lambda, \mu ; a, b) \\
& =\frac{1}{n+1}\left[\lambda f(a)+(1-\lambda) f\left(\frac{n}{n+1} a+\frac{1}{n+1} b\right)+(1-\mu) f\left(\frac{1}{n+1} a+\frac{n}{n+1} b\right)+\mu f(b)\right] .
\end{aligned}
$$

This completes the proof. 


\section{RESUlTS AND DisCUSSIONS}

In this section, we derive our main results.

Theorem 2. Let $f: I \rightarrow \mathbb{R}$ be differentiable function on $I^{o}, a, b \in I$ with $a<b$. If $\left|f^{\prime}\right|$ is h-convex function, $0 \leq \lambda, \mu \leq 1$ and $n \in \mathbb{N}^{*}$, then we have

$$
\begin{aligned}
& \left|\phi(n ; \lambda, \mu ; a, b)-\frac{1}{b-a}\left[\int_{a}^{\Omega_{1}} f(x) d x+\int_{\Omega_{2}}^{b} f(x) \mathrm{d} x\right]\right| \\
& \leq \frac{b-a}{(n+1)^{2}}\left[\alpha(t)\left|f^{\prime}(a)\right|+\beta(t)\left|f^{\prime}(b)\right|\right],
\end{aligned}
$$

where

$$
\alpha(t)=\int_{0}^{1}\left[(1-\lambda-t) h\left(\frac{n+t}{n+1}\right)+(\mu-t)\left(\frac{t}{n+1}\right)\right] \mathrm{d} t
$$

and

$$
\beta(t)=\int_{0}^{1}\left[(1-\lambda-t) h\left(\frac{1-t}{n+1}\right)+(\mu-t)\left(\frac{n+1-t}{n+1}\right)\right] \mathrm{d} t .
$$

Proof. Using Lemma 1, we have

$$
\begin{aligned}
& \left|\phi(n ; \lambda, \mu ; a, b)-\frac{1}{b-a}\left[\int_{a}^{\Omega_{1}} f(x) \mathrm{d} x+\int_{\Omega_{2}}^{b} f(x) \mathrm{d} x\right]\right| \\
& =\left|\frac{b-a}{(n+1)^{2}} \int_{0}^{1}\left[(1-\lambda-t) f^{\prime}\left(\frac{n+t}{n+1} a+\frac{1-t}{n+1} b\right)+(\mu-t) f^{\prime}\left(\frac{t}{n+1} a+\frac{n+1-t}{n+1} b\right)\right] \mathrm{d} t\right| .
\end{aligned}
$$

Take

$$
\begin{aligned}
I & =\left|\phi(n ; \lambda, \mu ; a, b)-\frac{1}{b-a}\left[\int_{a}^{\Omega_{1}} f(x) \mathrm{d} x+\int_{\Omega_{2}}^{b} f(x) \mathrm{d} x\right]\right| \\
& \leq \frac{b-a}{(n+1)^{2}} \int_{0}^{1}\left[|1-\lambda-t|\left|f^{\prime}\left(\frac{n+t}{n+1} a+\frac{1-t}{n+1} b\right)\right|+|\mu-t|\left|f^{\prime}\left(\frac{t}{n+1} a+\frac{n+1-t}{n+1} b\right)\right|\right] \mathrm{d} t .
\end{aligned}
$$

Since $\left|f^{\prime}\right|$ is an $h$-convex function, we have

$$
\begin{aligned}
& I \leq \frac{b-a}{(n+1)^{2}} \int_{0}^{1}\left[|1-\lambda-t|\left\{h\left(\frac{n+t}{n+1}\right)\left|f^{\prime}(a)\right|+h\left(\frac{1-t}{n+1}\right)\left|f^{\prime}(b)\right|\right\}\right. \\
&\left.+|\mu-t|\left\{h\left(\frac{t}{n+1}\right)\left|f^{\prime}(a)\right|+h\left(\frac{n+1-t}{n+1}\right)\left|f^{\prime}(b)\right|\right\}\right] \mathrm{d} t \\
&= \frac{b-a}{(n+1)^{2}} \int_{0}^{1}\left[\left\{|1-\lambda-t| h\left(\frac{n+t}{n+1}\right)+|\mu-t| h\left(\frac{t}{n+1}\right)\right\}\left|f^{\prime}(a)\right|\right. \\
&\left.+\left\{|1-\lambda-t| h\left(\frac{1-t}{n+1}\right)+|\mu-t| h\left(\frac{n+1-t}{n+1}\right)\right\}\left|f^{\prime}(b)\right|\right] \mathrm{d} t \\
&\left|\phi(n ; \lambda, \mu ; a, b)-\frac{1}{b-a}\left[\int_{a}^{\Omega_{1}} f(x) d x+\int_{\Omega_{2}}^{b} f(x) d x\right]\right|
\end{aligned}
$$




$$
\leq \frac{b-a}{(n+1)^{2}}\left[\alpha(t)\left|f^{\prime}(a)\right|+\beta(t)\left|f^{\prime}(b)\right|\right],
$$

where

$$
\alpha(t)=\int_{0}^{1}\left[|1-\lambda-t| h\left(\frac{n+t}{n+1}\right)+|\mu-t| h\left(\frac{t}{n+1}\right)\right] \mathrm{d} t
$$

and

$$
\beta(t)=\int_{0}^{1}\left[|1-\lambda-t| h\left(\frac{1-t}{n+1}\right)+|\mu-t| h\left(\frac{n+1-t}{n+1}\right)\right] \mathrm{d} t .
$$

This completes the proof.

Now we will discuss some special cases of the Theorem 2.

(i) When $h(t)=1$

Corollary 1. Let $f: I \rightarrow \mathbb{R}$ be differentiable function on $I^{o}, a, b \in I$ with $a<b$. If $\left|f^{\prime}\right|$ is $P$-function, $0 \leq \lambda, \mu \leq 1$ and $n \in \mathbb{N}^{*}$, then we have

$$
\begin{aligned}
& \left|\phi(n ; \lambda, \mu ; a, b)-\frac{1}{b-a}\left[\int_{a}^{\Omega_{1}} f(x) d x+\int_{\Omega_{2}}^{b} f(x) d x\right]\right| \\
& \leq \frac{b-a}{(n+1)^{2}}\left[\left(\lambda^{2}+\mu^{2}\right)-(\lambda+\mu)+1\right]\left(\left|f^{\prime}(a)\right|+\left|f^{\prime}(b)\right|\right) .
\end{aligned}
$$

(ii) When $h(t)=t$

Corollary 2. Let $f: I \rightarrow \mathbb{R}$ be differentiable function on $I^{o}, a, b \in I$ with $a<b$. If $\left|f^{\prime}\right|$ is classical convex function, $0 \leq \lambda, \mu \leq 1$ and $n \in \mathbb{N}^{*}$, then we have

$$
\begin{aligned}
& \left|\phi(n ; \lambda, \mu ; a, b)-\frac{1}{b-a}\left[\int_{a}^{\Omega_{1}} f(x) d x+\int_{\Omega_{2}}^{b} f(x) d x\right]\right| \\
& \leq \frac{b-a}{6(n+1)^{3}} \times\left[\left\{3(1+n)-3(1+2 n) \lambda-3 \mu+6(1+n) \lambda^{2}-2\left(\lambda^{3}-\mu^{3}\right)\right\}\left|f^{\prime}(a)\right|\right. \\
& \left.\quad+\left\{3(1+n)-3(1+2 n) \mu-3 \lambda+6(1+n) \mu^{2}-2\left(\mu^{3}-\lambda^{3}\right)\right\}\left|f^{\prime}(b)\right|\right] .
\end{aligned}
$$

(iii) When $h(t)=t^{s}$

Corollary 3. Let $f: I \rightarrow \mathbb{R}$ be differentiable function on $I^{o}, a, b \in I$ with $a<b$. If $\left|f^{\prime}\right|$ is Breckner type of s-convex function, $0 \leq \lambda, \mu \leq 1$ and $n \in \mathbb{N}^{*}$, then we have

$$
\begin{aligned}
& \left|\phi(n ; \lambda, \mu ; a, b)-\frac{1}{b-a}\left[\int_{a}^{\Omega_{1}} f(x) \mathrm{d} x+\int_{\Omega_{2}}^{b} f(x) \mathrm{d} x\right]\right| \\
& \leq \frac{b-a}{(n+1)^{s+2}(s+1)(s+2)} \times\left[\left\{(s+2)(\lambda-1) n^{s+1}+2(1-\lambda+n)^{s+2}\right.\right. \\
& \left.\quad+(s+2)(n+1)^{s+1} \lambda-n^{s+2}-(n+1)^{s+2}+2 \mu^{s+2}+(s+2)(1-\mu)-1\right\}\left|f^{\prime}(a)\right|^{q}
\end{aligned}
$$




$$
\begin{aligned}
& +\left\{2 \lambda^{s+2}+(s+2)(1-\lambda)-1+(s+2)(\mu-1) n^{s+1}+2(1-\mu+n)^{s+2}\right. \\
& \left.\left.+(s+2)(n+1)^{s+1} \mu-n^{s+2}-(n+1)^{s+2}\right\}\left|f^{\prime}(b)\right|^{q}\right] .
\end{aligned}
$$

(iv) When $h(t)=t^{-s}$

Corollary 4. Let $f: I \rightarrow \mathbb{R}$ be differentiable function on $I^{o}, a, b \in I$ with $a<b$. If $\left|f^{\prime}\right|$ is Godunova-Levin-Dragomir type of s-convex function, $0 \leq \lambda, \mu \leq 1$ and $n \in \mathbb{N}^{*}$, then we have

$$
\begin{aligned}
& \left|\phi(n ; \lambda, \mu ; a, b)-\frac{1}{b-a}\left[\int_{a}^{\Omega_{1}} f(x) \mathrm{d} x+\int_{\Omega_{2}}^{b} f(x) \mathrm{d} x\right]\right| \\
& \leq \frac{b-a}{(1-s)(2-s)(n+1)^{2-s}} \times\left[\left\{(2-s)(\lambda-1) n^{1-s}+2(1-\lambda+n)^{2-s}\right.\right. \\
& \left.\quad+(2-s)(n+1)^{1-s} \lambda-n^{2-s}-(n+1)^{2-s}+2 \mu^{2-s}+(2-s)(1-\mu)-1\right\}\left|f^{\prime}(a)\right| \\
& \quad+\left\{2 \lambda^{2-s}+(2-s)(1-\lambda)-1+(2-s)(\mu-1) n^{1-s}+2(1-\mu+n)^{2-s}\right. \\
& \left.\left.\quad+(2-s)(n+1)^{1-s} \mu-n^{2-s}-(n+1)^{2-s}\right\}\left|f^{\prime}(b)\right|\right] .
\end{aligned}
$$

(v) When $h(t)=t(1-t)$

Corollary 5. Let $f: I \rightarrow \mathbb{R}$ be differentiable function on $I^{o}, a, b \in I$ with $a<b$. If $\left|f^{\prime}\right|$ is tgs-convex function, $0 \leq \lambda, \mu \leq 1$ and $n \in \mathbb{N}^{*}$, then we have

$$
\begin{aligned}
& \left|\phi(n ; \lambda, \mu ; a, b)-\frac{1}{b-a}\left[\int_{a}^{\Omega_{1}} f(x) d x+\int_{\Omega_{2}}^{b} f(x) d x\right]\right| \\
& \leq\left[\frac{(b-a)\left(\left|f^{\prime}(a)\right|+\left|f^{\prime}(a)\right|\right)}{12(n+1)^{4}}\right] \times\left[2(1+3 n)(\mu-\lambda)+4(1+n)\left(\mu^{3}+\lambda^{3}\right)-2\left(\mu^{4}+\lambda^{4}\right)\right] .
\end{aligned}
$$

Theorem 3. Let $f: I \rightarrow \mathbb{R}$ be differentiable function on $I^{o}$, $a, b \in I$ with $a<b$. If $\left|f^{\prime}\right|^{q}$ is h-convex function, $0 \leq \lambda, \mu \leq 1$ and $n \in \mathbb{N}^{*}$, then for $\frac{1}{p}+\frac{1}{q}=1, q>1$, we have

$$
\begin{aligned}
& \left|\phi(n ; \lambda, \mu ; a, b)-\frac{1}{b-a}\left[\int_{a}^{\Omega_{1}} f(x) d x+\int_{\Omega_{2}}^{b} f(x) \mathrm{d} x\right]\right| \\
& \leq \frac{b-a}{(n+1)^{2}}\left(\frac{1}{p+1}\right)^{\frac{1}{p}}\left[\left(\lambda^{p+1}+(1-\lambda)^{p+1}\right)^{\frac{1}{p}} K_{1}+\left(\mu^{p+1}+(1-\mu)^{p+1}\right)^{\frac{1}{p}} K_{2}\right],
\end{aligned}
$$

where

$$
K_{1}=\left(\left|f^{\prime}(a)\right|^{q} \int_{0}^{1} h\left(\frac{n+t}{n+1}\right) \mathrm{d} t+\left|f^{\prime}(b)\right|^{q} \int_{0}^{1} h\left(\frac{1-t}{n+1}\right) \mathrm{d} t\right)^{\frac{1}{q}}
$$

and

$$
K_{2}=\left(\left|f^{\prime}(a)\right|^{q} \int_{0}^{1} h\left(\frac{t}{n+1}\right) \mathrm{d} t+\left|f^{\prime}(b)\right|^{q} \int_{0}^{1} h\left(\frac{n+1-t}{n+1}\right) \mathrm{d} t\right)^{\frac{1}{q}}
$$


Proof. Using Lemma 1, we have

$$
\begin{aligned}
& \left|\phi(n ; \lambda, \mu ; a, b)-\frac{1}{b-a}\left[\int_{a}^{\Omega_{1}} f(x) \mathrm{d} x+\int_{\Omega_{2}}^{b} f(x) \mathrm{d} x\right]\right| \\
& =\left|\frac{b-a}{(n+1)^{2}} \int_{0}^{1}\left[(1-\lambda-t) f^{\prime}\left(\frac{n+t}{n+1} a+\frac{1-t}{n+1} b\right)+(\mu-t) f^{\prime}\left(\frac{t}{n+1} a+\frac{n+1-t}{n+1} b\right)\right] \mathrm{d} t\right| .
\end{aligned}
$$

Now using the property of modulus, we have

$$
\begin{aligned}
& \left|\phi(n ; \lambda, \mu ; a, b)-\frac{1}{b-a}\left[\int_{a}^{\Omega_{1}} f(x) \mathrm{d} x+\int_{\Omega_{2}}^{b} f(x) \mathrm{d} x\right]\right| \\
& \leq \frac{b-a}{(n+1)^{2}}\left[\int_{0}^{1}|1-\lambda-t|\left|f^{\prime}\left(\frac{n+t}{n+1} a+\frac{1-t}{n+1} b\right)\right| \mathrm{d} t\right. \\
& \left.\quad+\int_{0}^{1}|\mu-t|\left|f^{\prime}\left(\frac{t}{n+1} a+\frac{n+1-t}{n+1} b\right)\right| \mathrm{d} t\right] \\
& =\frac{b-a}{(n+1)^{2}}\left[I_{1}+I_{2}\right] .
\end{aligned}
$$

Using Hölder's inequality, we have

$$
\begin{aligned}
& \int_{0}^{1}|1-\lambda-t|\left|f^{\prime}\left(\frac{n+t}{n+1} a+\frac{1-t}{n+1} b\right)\right| \mathrm{d} t \\
& \leq\left(\int_{0}^{1}|1-\lambda-t|^{p} \mathrm{~d} t\right)^{\frac{1}{p}}\left(\int_{0}^{1}\left|f^{\prime}\left(\frac{n+t}{n+1} a+\frac{1-t}{n+1} b\right)\right|^{q} \mathrm{~d} t\right)^{\frac{1}{q}},
\end{aligned}
$$

where $\frac{1}{p}+\frac{1}{q}=1$.

$$
\int_{0}^{1}|1-\lambda-t|^{p} \mathrm{~d} t=\frac{(1-\lambda)^{p+1}+(\lambda)^{p+1}}{p+1} .
$$

Since $\left|f^{\prime}\right|^{q}$ is $h$-convex, then

$$
\begin{aligned}
& \int_{0}^{1}\left|f^{\prime}\left(\frac{n+t}{n+1} a+\frac{1-t}{n+1} b\right)\right|^{q} \mathrm{~d} t \\
& \leq \int_{0}^{1} h\left(\frac{n+t}{n+1}\right)\left|f^{\prime}(a)\right|^{q} \mathrm{~d} t+\int_{0}^{1} h\left(\frac{1-t}{n+1}\right)\left|f^{\prime}(b)\right|^{q} \mathrm{~d} t .
\end{aligned}
$$

Using (3.6) and (3.7) in (3.5), we have

$$
\begin{aligned}
& \int_{0}^{1}|1-\lambda-t|\left|f^{\prime}\left(\frac{n+t}{n+1} a+\frac{1-t}{n+1} b\right)\right| \mathrm{d} t \\
& \leq\left(\frac{(1-\lambda)^{p+1}+(\lambda)^{p+1}}{p+1}\right)^{\frac{1}{p}}\left(\int_{0}^{1} h\left(\frac{n+t}{n+1}\right)\left|f^{\prime}(a)\right|^{q} \mathrm{~d} t+\int_{0}^{1} h\left(\frac{1-t}{n+1}\right)\left|f^{\prime}(b)\right|^{q} \mathrm{~d} t\right)^{\frac{1}{q}} .
\end{aligned}
$$


Now

$$
\int_{0}^{1}|\mu-t|\left|f^{\prime}\left(\frac{t}{n+1} a+\frac{n+1-t}{n+1} b\right)\right| \mathrm{d} t
$$

Using Hölder's inequality, we have

$$
\begin{aligned}
& \int_{0}^{1}|\mu-t|\left|f^{\prime}\left(\frac{t}{n+1} a+\frac{n+1-t}{n+1} b\right)\right| \mathrm{d} t \\
& \leq\left(\int_{0}^{1}|\mu-t|^{p} \mathrm{~d} t\right)^{\frac{1}{p}}\left(\int_{0}^{1}\left|f^{\prime}\left(\frac{t}{n+1} a+\frac{n+1-t}{n+1} b\right)\right|^{q} \mathrm{~d} t\right)^{\frac{1}{q}}
\end{aligned}
$$

where $\frac{1}{p}+\frac{1}{q}=1$.

$$
\int_{0}^{1}|\mu-t|^{p} \mathrm{~d} t=\frac{(1-\mu)^{p+1}+(\mu)^{p+1}}{p+1}
$$

Since $\left|f^{\prime}\right|^{q}$ is $h$-convex, then

$$
\begin{aligned}
& \int_{0}^{1}\left|f^{\prime}\left(\frac{t}{n+1} a+\frac{n+1-t}{n+1} b\right)\right|^{q} \mathrm{~d} t \\
& \leq \int_{0}^{1} h\left(\frac{t}{n+1}\right)\left|f^{\prime}(a)\right|^{q} \mathrm{~d} t+\int_{0}^{1} h\left(\frac{n+1-t}{n+1}\right)\left|f^{\prime}(b)\right|^{q} \mathrm{~d} t .
\end{aligned}
$$

Using (3.10) and (3.11) in (3.9), we have

$$
\begin{aligned}
& \int_{0}^{1}|\mu-t|\left|f^{\prime}\left(\frac{t}{n+1} a+\frac{n+1-t}{n+1} b\right)\right| \mathrm{d} t \\
& \leq\left(\frac{(1-\mu)^{p+1}+(\mu)^{p+1}}{p+1}\right)^{\frac{1}{p}}\left(\int_{0}^{1} h\left(\frac{t}{n+1}\right)\left|f^{\prime}(a)\right|^{q} \mathrm{~d} t+\int_{0}^{1} h\left(\frac{n+1-t}{n+1}\right)\left|f^{\prime}(b)\right|^{q} \mathrm{~d} t\right)^{\frac{1}{q}} .
\end{aligned}
$$

Now using (3.8) and (3.12) in (3.4), we have

$$
\begin{aligned}
& \left|\phi(n ; \lambda, \mu ; a, b)-\frac{1}{b-a}\left[\int_{a}^{\Omega_{1}} f(x) \mathrm{d} x+\int_{\Omega_{2}}^{b} f(x) \mathrm{d} x\right]\right| \\
& \leq \frac{b-a}{(n+1)^{2}}\left[\left(\frac{\lambda^{p+1}+(1-\lambda)^{p+1}}{p+1}\right)^{\frac{1}{p}} K_{1}+\left(\frac{\mu^{p+1}+(1-\mu)^{p+1}}{p+1}\right)^{\frac{1}{p}} K_{2}\right],
\end{aligned}
$$

where

$$
K_{1}=\left(\int_{0}^{1} h\left(\frac{n+t}{n+1}\right)\left|f^{\prime}(a)\right|^{q} \mathrm{~d} t+\int_{0}^{1} h\left(\frac{1-t}{n+1}\right)\left|f^{\prime}(b)\right|^{q} \mathrm{~d} t\right)^{\frac{1}{q}}
$$

and

$$
K_{2}=\left(\int_{0}^{1} h\left(\frac{t}{n+1}\right)\left|f^{\prime}(a)\right|^{q} \mathrm{~d} t+\int_{0}^{1} h\left(\frac{n+1-t}{n+1}\right)\left|f^{\prime}(b)\right|^{q} \mathrm{~d} t\right)^{\frac{1}{q}}
$$


This completes the proof.

Now we will discuss some special cases of Theorem 3

(i) When $h(t)=1$

Corollary 6. Let $f: I \rightarrow \mathbb{R}$ be differentiable function on $I^{o}, a, b \in I$ with $a<b$. If $\left|f^{\prime}\right|^{q}$ is $P$-function, $0 \leq \lambda, \mu \leq 1$ and $n \in \mathbb{N}^{*}$, then we have

$$
\begin{aligned}
& \left|\phi(n ; \lambda, \mu ; a, b)-\frac{1}{b-a}\left[\int_{a}^{\Omega_{1}} f(x) d x+\int_{\Omega_{2}}^{b} f(x) \mathrm{d} x\right]\right| \\
& \leq \frac{b-a}{(n+1)^{2}}\left(\left(\frac{\lambda^{p+1}+(1-\lambda)^{p+1}}{p+1}\right)^{\frac{1}{p}}+\left(\frac{\mu^{p+1}+(1-\mu)^{p+1}}{p+1}\right)^{\frac{1}{p}}\right) \times\left(\left|f^{\prime}(a)\right|^{q}+\left|f^{\prime}(b)\right|^{q}\right)^{\frac{1}{q}} .
\end{aligned}
$$

(ii) When $h(t)=t$

Corollary 7. Let $f: I \rightarrow \mathbb{R}$ be differentiable function on $I^{o}, a, b \in I$ with $a<b$. If $\left|f^{\prime}\right|^{q}$ is classical convex function, $0 \leq \lambda, \mu \leq 1$ and $n \in \mathbb{N}^{*}$, then we have

$$
\begin{aligned}
& \left|\phi(n ; \lambda, \mu ; a, b)-\frac{1}{b-a}\left[\int_{a}^{\Omega_{1}} f(x) d x+\int_{\Omega_{2}}^{b} f(x) \mathrm{d} x\right]\right| \\
& \leq \frac{b-a}{(n+1)^{2}}\left[\left(\frac{\lambda^{p+1}+(1-\lambda)^{p+1}}{p+1}\right)^{\frac{1}{p}}\left(\frac{(2 n+1)\left|f^{\prime}(a)\right|^{q}+\left|f^{\prime}(b)\right|^{q}}{2(n+1)}\right)^{\frac{1}{q}}\right. \\
& \left.\quad+\left(\frac{\mu^{p+1}+(1-\mu)^{p+1}}{p+1}\right)^{\frac{1}{p}}\left(\frac{\left|f^{\prime}(a)\right|^{q}+(2 n+1)\left|f^{\prime}(b)\right|^{q}}{2(n+1)}\right)^{\frac{1}{q}}\right] .
\end{aligned}
$$

(iii) When $h(t)=t^{s}$

Corollary 8. Let $f: I \rightarrow \mathbb{R}$ be differentiable function on $I^{o}, a, b \in I$ with $a<b$. If $\left|f^{\prime}\right|^{q}$ is Breckner type of s-convex function, $0 \leq \lambda, \mu \leq 1$ and $n \in \mathbb{N}^{*}$, then we have

$$
\begin{aligned}
& \left|\phi(n ; \lambda, \mu ; a, b)-\frac{1}{b-a}\left[\int_{a}^{\Omega_{1}} f(x) d x+\int_{\Omega_{2}}^{b} f(x) \mathrm{d} x\right]\right| \\
& \leq \frac{b-a}{(n+1)^{2}}\left[\left(\frac{\lambda^{p+1}+(1-\lambda)^{p+1}}{p+1}\right)^{\frac{1}{p}}\left(\frac{\left((n+1)^{1+s}-n^{1+s}\right)\left|f^{\prime}(a)\right|^{q}+\left|f^{\prime}(b)\right|^{q}}{(1+s)(n+1)^{s}}\right)^{\frac{1}{q}}\right. \\
& \left.\quad+\left(\frac{\mu^{p+1}+(1-\mu)^{p+1}}{p+1}\right)^{\frac{1}{p}}\left(\frac{\left|f^{\prime}(a)\right|^{q}+\left((n+1)^{1+s}-n^{1+s}\right)\left|f^{\prime}(b)\right|^{q}}{(1+s)(n+1)^{s}}\right)^{\frac{1}{q}}\right] .
\end{aligned}
$$

(iv) When $h(t)=t^{-s}$ 
Corollary 9. Let $f: I \rightarrow \mathbb{R}$ be differentiable function on $I^{o}, a, b \in I$ with $a<b$. If $\left|f^{\prime}\right|^{q}$ is Godunova-Levin-Dragomir type of s-convex function, $0 \leq \lambda, \mu \leq 1$ and $n \in \mathbb{N}^{*}$, then we have

$$
\begin{aligned}
& \left|\phi(n ; \lambda, \mu ; a, b)-\frac{1}{b-a}\left[\int_{a}^{\Omega_{1}} f(x) d x+\int_{\Omega_{2}}^{b} f(x) \mathrm{d} x\right]\right| \\
& \leq \frac{b-a}{(n+1)^{2}}\left[\left(\frac{\lambda^{p+1}+(1-\lambda)^{p+1}}{p+1}\right)^{\frac{1}{p}}\left(\frac{\left((n+1)^{1-s}-n^{1-s}\right)\left|f^{\prime}(a)\right|^{q}+\left|f^{\prime}(b)\right|^{q}}{(1-s)(n+1)^{-s}}\right)^{\frac{1}{q}}\right. \\
& \left.\quad+\left(\frac{\mu^{p+1}+(1-\mu)^{p+1}}{p+1}\right)^{\frac{1}{p}}\left(\frac{\left|f^{\prime}(a)\right|^{q}+\left((n+1)^{1-s}-n^{1-s}\right)\left|f^{\prime}(b)\right|^{q}}{(1-s)(n+1)^{-s}}\right)^{\frac{1}{q}}\right] .
\end{aligned}
$$

(v) When $h(t)=t(1-t)$

Corollary 10. Let $f: I \rightarrow \mathbb{R}$ be differentiable function on $I^{o}, a, b \in I$ with $a<b$. If $\left|f^{\prime}\right|^{q}$ is tgs-convex function, $0 \leq \lambda, \mu \leq 1$ and $n \in \mathbb{N}^{*}$, then we have

$$
\begin{aligned}
& \left|\phi(n ; \lambda, \mu ; a, b)-\frac{1}{b-a}\left[\int_{a}^{\Omega_{1}} f(x) d x+\int_{\Omega_{2}}^{b} f(x) \mathrm{d} x\right]\right| \\
& \leq \frac{b-a}{(n+1)^{2}}\left[\left(\frac{\lambda^{p+1}+(1-\lambda)^{p+1}}{p+1}\right)^{\frac{1}{p}}+\left(\frac{\mu^{p+1}+(1-\mu)^{p+1}}{p+1}\right)^{\frac{1}{p}}\right] \\
& \quad \times\left[\frac{(3 n+1)\left(\left|f^{\prime}(a)\right|^{q}+\left|f^{\prime}(b)\right|^{q}\right)}{6(n+1)^{2}}\right]^{\frac{1}{q}} .
\end{aligned}
$$

Theorem 4. Let $f: I \rightarrow \mathbb{R}$ be differentiable function on $I^{o}, a, b \in I$ with $a<b$. If $\left|f^{\prime}\right|^{q}$ is h-convex function, $0 \leq \lambda, \mu \leq 1$ and $n \in \mathbb{N}^{*}$, then we have

$$
\begin{aligned}
& \left|\phi(n ; \lambda, \mu ; a, b)-\frac{1}{b-a}\left[\int_{a}^{\Omega_{1}} f(x) \mathrm{d} x+\int_{\Omega_{2}}^{b} f(x) \mathrm{d} x\right]\right| \\
& \leq \frac{b-a}{(n+1)^{2}}\left[\left(\frac{1}{2}-\lambda+\lambda^{2}\right)^{1-\frac{1}{q}} K_{1}^{\frac{1}{q}}+\left(\frac{1}{2}-\mu+\mu^{2}\right)^{1-\frac{1}{q}} K_{2}^{\frac{1}{q}}\right],
\end{aligned}
$$

where

$$
K_{1}^{*}=\int_{0}^{1}|1-\lambda-t|\left(h\left(\frac{n+t}{n+1}\right)\left|f^{\prime}(a)\right|^{q}+h\left(\frac{1-t}{n+1}\right)\left|f^{\prime}(b)\right|^{q}\right) \mathrm{d} t
$$

and

$$
K_{2}^{*}=\int_{0}^{1}|\mu-t|\left(h\left(\frac{t}{n+1}\right)\left|f^{\prime}(a)\right|^{q}+h\left(\frac{n+1-t}{n+1}\right)\left|f^{\prime}(b)\right|^{q}\right) \mathrm{d} t .
$$

Proof. Using Lemma 1, we have

$$
\left|\phi(n ; \lambda, \mu ; a, b)-\frac{1}{b-a}\left[\int_{a}^{\Omega_{1}} f(x) \mathrm{d} x+\int_{\Omega_{2}}^{b} f(x) \mathrm{d} x\right]\right|
$$


$=\left|\frac{b-a}{(n+1)^{2}} \int_{0}^{1}\left[(1-\lambda-t) f^{\prime}\left(\frac{n+t}{n+1} a+\frac{1-t}{n+1} b\right)+(\mu-t) f^{\prime}\left(\frac{t}{n+1} a+\frac{n+1-t}{n+1} b\right)\right] \mathrm{d} t\right|$.

Now using the property of modulus, we have

$$
\begin{aligned}
& \left|\phi(n ; \lambda, \mu ; a, b)-\frac{1}{b-a}\left[\int_{a}^{\Omega_{1}} f(x) \mathrm{d} x+\int_{\Omega_{2}}^{b} f(x) \mathrm{d} x\right]\right| \\
& \leq \frac{b-a}{(n+1)^{2}}\left[\int_{0}^{1}|1-\lambda-t|\left|f^{\prime}\left(\frac{n+t}{n+1} a+\frac{1-t}{n+1} b\right)\right| \mathrm{d} t\right. \\
& \left.\quad+\int_{0}^{1}|\mu-t|\left|f^{\prime}\left(\frac{t}{n+1} a+\frac{n+1-t}{n+1} b\right)\right| \mathrm{d} t\right] .
\end{aligned}
$$

Using Hölder's integral inequality and $h$-convexity, we have

$$
\begin{aligned}
& \int_{0}^{1}|1-\lambda-t|\left|f^{\prime}\left(\frac{n+t}{n+1} a+\frac{1-t}{n+1} b\right)\right| \mathrm{d} t \\
& \leq\left(\int_{0}^{1}|1-\lambda-t| \mathrm{d} t\right)^{1-\frac{1}{q}} \\
& \quad \times\left(\int_{0}^{1}|1-\lambda-t|\left(h\left(\frac{n+t}{n+1}\right)\left|f^{\prime}(a)\right|^{q}+h\left(\frac{1-t}{n+1}\right)\left|f^{\prime}(b)\right|^{q}\right) \mathrm{d} t\right)^{\frac{1}{q}} \\
& =\left(\frac{1}{2}-\lambda+\lambda^{2}\right)^{1-\frac{1}{q}} K_{1}^{* \frac{1}{q}}
\end{aligned}
$$

Now

$$
\begin{aligned}
& \int_{0}^{1}|\mu-t|\left|f^{\prime}\left(\frac{t}{n+1} a+\frac{n+1-t}{n+1} b\right)\right| \mathrm{d} t \\
& \leq\left(\int_{0}^{1}|\mu-t| \mathrm{d} t\right)^{1-\frac{1}{q}} \times\left(\int_{0}^{1}|\mu-t|\left(h\left(\frac{t}{n+1}\right)\left|f^{\prime}(a)\right|^{q}+h\left(\frac{n+1-t}{n+1}\right)\left|f^{\prime}(b)\right|^{q}\right) \mathrm{d} t\right)^{\frac{1}{q}} \\
& =\left(\frac{1}{2}-\mu+\mu^{2}\right)^{1-\frac{1}{q}} K_{2}^{* \frac{1}{q}} .
\end{aligned}
$$

Using above two inequalities in (3.14), we have

$$
\begin{aligned}
& \left|\phi(n ; \lambda, \mu ; a, b)-\frac{1}{b-a}\left[\int_{a}^{\Omega_{1}} f(x) \mathrm{d} x+\int_{\Omega_{2}}^{b} f(x) \mathrm{d} x\right]\right| \\
& \leq \frac{b-a}{(n+1)^{2}}\left[\left(\frac{1}{2}-\lambda+\lambda^{2}\right)^{1-\frac{1}{q}} K_{1} \frac{1}{q}+\left(\frac{1}{2}-\mu+\mu^{2}\right)^{1-\frac{1}{q}} K_{2}^{\frac{1}{q}}\right],
\end{aligned}
$$

where

$$
K_{1}=\int_{0}^{1}|1-\lambda-t|\left(h\left(\frac{n+t}{n+1}\right)\left|f^{\prime}(a)\right|^{q}+h\left(\frac{1-t}{n+1}\right)\left|f^{\prime}(b)\right|^{q}\right) \mathrm{d} t
$$


and

$$
K_{2}=\int_{0}^{1}|\mu-t|\left(h\left(\frac{t}{n+1}\right)\left|f^{\prime}(a)\right|^{q}+h\left(\frac{n+1-t}{n+1}\right)\left|f^{\prime}(b)\right|^{q}\right) \mathrm{d} t .
$$

This completes the proof.

Now we will discuss some cases of Theorem 4.

(i) When $h(t)=1$

Corollary 11. Let $f: I \rightarrow \mathbb{R}$ be differentiable function on $I^{o}, a, b \in I$ with $a<b$. If $\left|f^{\prime}\right|^{q}$ is $P$-convex function, $0 \leq \lambda, \mu \leq 1$ and $n \in \mathbb{N}^{*}$, then we have

$$
\begin{aligned}
& \left|\phi(n ; \lambda, \mu ; a, b)-\frac{1}{b-a}\left[\int_{a}^{\Omega_{1}} f(x) \mathrm{d} x+\int_{\Omega_{2}}^{b} f(x) \mathrm{d} x\right]\right| \\
& \leq \frac{b-a}{(n+1)^{2}}\left(\lambda^{2}+\mu^{2}-(\lambda+\mu)+1\right)\left(\left|f^{\prime}(a)\right|^{q}+\left|f^{\prime}(b)\right|^{q}\right)^{\frac{1}{q}} .
\end{aligned}
$$

(ii) When $h(t)=t$

Corollary 12. Let $f: I \rightarrow \mathbb{R}$ be differentiable function on $I^{o}, a, b \in I$ with $a<b$. If $\left|f^{\prime}\right|^{q}$ is classical convex function, $0 \leq \lambda, \mu \leq 1$ and $n \in \mathbb{N}^{*}$, then we have

$$
\begin{aligned}
& \left|\phi(n ; \lambda, \mu ; a, b)-\frac{1}{b-a}\left[\int_{a}^{\Omega_{1}} f(x) \mathrm{d} x+\int_{\Omega_{2}}^{b} f(x) \mathrm{d} x\right]\right| \\
& \leq \frac{b-a}{(n+1)^{2}}\left[( \frac { 1 } { 2 } - \lambda + \lambda ^ { 2 } ) ^ { 1 - \frac { 1 } { q } } \left\{\frac{\left|f^{\prime}(a)\right|^{q}}{6(n+1)}((3 n+1)-(6 n+3) \lambda\right.\right. \\
& \left.\left.\quad+6(n+1) \lambda^{2}-2 \lambda^{3}\right)+\frac{\left|f^{\prime}(b)\right|^{q}}{6(n+1)}\left(2-3 \lambda+2 \lambda^{3}\right)\right\}^{\frac{1}{q}} \\
& \quad+\left(\frac{1}{2}-\mu+\mu^{2}\right)^{1-\frac{1}{q}}\left\{\frac{\left|f^{\prime}(a)\right|^{q}}{6(n+1)}\left(2-3 \mu+2 \mu^{3}\right)\right. \\
& \left.\left.\quad+\frac{\left|f^{\prime}(b)\right|^{q}}{6(n+1)}\left((3 n+1)-(6 n+3) \mu+6(n+1) \mu^{2}-2 \mu^{3}\right)\right\}^{\frac{1}{q}}\right] .
\end{aligned}
$$

(iii) When $h(t)=t^{s}$

Corollary 13. Let $f: I \rightarrow \mathbb{R}$ be differentiable function on $I^{o}, a, b \in I$ with $a<b$. If $\left|f^{\prime}\right|^{q}$ is Breckner type of s-convex function, $0 \leq \lambda, \mu \leq 1$ and $n \in \mathbb{N}^{*}$, then we have

$$
\begin{aligned}
& \left|\phi(n ; \lambda, \mu ; a, b)-\frac{1}{b-a}\left[\int_{a}^{\Omega_{1}} f(x) \mathrm{d} x+\int_{\Omega_{2}}^{b} f(x) \mathrm{d} x\right]\right| \\
& \leq \frac{b-a}{(n+1)^{2}} \times\left[( \frac { 1 } { 2 } - \lambda + \lambda ^ { 2 } ) ^ { 1 - \frac { 1 } { q } } \left\{\frac{\left|f^{\prime}(a)\right|^{q}}{(n+1)^{s}}\left(\frac{(s+2)(\lambda-1) n^{s+1}+2(1-\lambda+n)^{s+2}}{(s+1)(s+2)}\right)\right.\right.
\end{aligned}
$$




$$
\begin{aligned}
& \left.\left.+\frac{(s+2)(n+1)^{s+1} \lambda-n^{s+2}-(n+1)^{s+2}}{(s+1)(s+2)}\right)+\frac{\left|f^{\prime}(b)\right|^{q}}{(n+1)^{s}}\left(\frac{2 \lambda^{s+2}+(s+2)(1-\lambda)-1}{(s+1)(s+2)}\right)\right\}^{\frac{1}{q}} \\
& +\left(\frac{1}{2}-\mu+\mu^{2}\right)^{1-\frac{1}{q}}\left\{\frac{\left|f^{\prime}(a)\right|^{q}}{(n+1)^{s}}\left(\frac{2 \mu^{s+2}+(s+2)(1-\mu)-1}{(s+1)(s+2)}\right)\right. \\
& +\frac{\left|f^{\prime}(b)\right|^{q}}{(n+1)^{s}}\left(\frac{(s+2)(\mu-1) n^{s+1}+(1-\mu+n)^{s+2}}{(s+1)(s+2)}\right. \\
& \left.\left.+\frac{(s+2)(n+1)^{s+1} \mu-n^{s+2}-(n+1)^{s+2}}{(s+1)(s+2)}\right)\right\}^{\frac{1}{q}} .
\end{aligned}
$$

(iv) When $h(t)=t^{-s}$

Corollary 14. Let $f: I \rightarrow \mathbb{R}$ be differentiable function on $I^{o}, a, b \in I$ with $a<b$. If $\left|f^{\prime}\right|^{q}$ is Godunova-Levin-Dragomir type of s-convex function, $0 \leq \lambda, \mu \leq 1$ and $n \in \mathbb{N}^{*}$, then we have

$$
\begin{aligned}
& \left|\phi(n ; \lambda, \mu ; a, b)-\frac{1}{b-a}\left[\int_{a}^{\Omega_{1}} f(x) \mathrm{d} x+\int_{\Omega_{2}}^{b} f(x) \mathrm{d} x\right]\right| \\
& \leq \frac{b-a}{(n+1)^{2}} \times\left[( \frac { 1 } { 2 } - \lambda + \lambda ^ { 2 } ) ^ { 1 - \frac { 1 } { q } } \left\{\frac{\left|f^{\prime}(a)\right|^{q}}{(n+1)^{-s}}\left(\frac{(2-s)(\lambda-1) n^{1-s}+2(1-\lambda+n)^{2-s}}{(1-s)(2-s)}\right)\right.\right. \\
& \left.\left.\quad+\frac{(2-s)(n+1)^{1-s} \lambda-n^{2-s}-(n+1)^{2-s}}{(1-s)(2-s)}\right)+\frac{\left|f^{\prime}(b)\right|^{q}}{(n+1)^{-s}}\left(\frac{2 \lambda^{2-s}+(2-s)(1-\lambda)-1}{(1-s)(2-s)}\right)\right\}^{\frac{1}{q}} \\
& \quad+\left(\frac{1}{2}-\mu+\mu^{2}\right)^{1-\frac{1}{q}}\left\{\frac{\left|f^{\prime}(a)\right|^{q}}{(n+1)^{-s}}\left(\frac{2 \mu^{2-s}+(2-s)(1-\mu)-1}{(1-s)(2-s)}\right)\right. \\
& \quad+\frac{\left|f^{\prime}(b)\right|^{q}}{(n+1)^{-s}}\left(\frac{(2-s)(\mu-1) n^{1-s}+(1-\mu+n)^{2-s}}{(1-s)(2-s)}\right. \\
& \left.\left.\left.\quad+\frac{(2-s)(n+1)^{1-s} \mu-n^{2-s}-(n+1)^{2-s}}{(1-s)(2-s)}\right)\right\}^{\frac{1}{q}}\right] .
\end{aligned}
$$

(v) When $h(t)=t(1-t)$

Corollary 15. Let $f: I \rightarrow \mathbb{R}$ be differentiable function on $I^{o}, a, b \in I$ with $a<b$. If $\left|f^{\prime}\right|^{q}$ is tgs-convex function, $0 \leq \lambda, \mu \leq 1$ and $n \in \mathbb{N}^{*}$, then we have

$$
\begin{aligned}
& \left|\phi(n ; \lambda, \mu ; a, b)-\frac{1}{b-a}\left[\int_{a}^{\Omega_{1}} f(x) \mathrm{d} x+\int_{\Omega_{2}}^{b} f(x) \mathrm{d} x\right]\right| \\
& \leq \frac{b-a}{(n+1)^{2}}\left(\frac{\left|f^{\prime}(a)\right|^{q}+\left|f^{\prime}(b)\right|^{q}}{(n+1)^{2}}\right)^{\frac{1}{q}}\left[\left(\frac{1}{2}-\lambda+\lambda^{2}\right)^{1-\frac{1}{q}}\right. \\
& \quad\left(\frac{(1-\lambda)^{2}\left(1+4 n+2 n \lambda-\lambda^{2}\right)}{6}+\frac{6 n \lambda+2 \lambda-4 n-1}{12}\right)^{\frac{1}{q}}
\end{aligned}
$$




$$
\left.+\left(\frac{1}{2}-\mu+\mu^{2}\right)^{1-\frac{1}{q}}\left(\frac{\mu^{3}(2+2 n-\mu)}{6}+\frac{6 n \mu+2 \mu-4 n-1}{12}\right)^{\frac{1}{q}}\right] .
$$

Theorem 5. Let $f: I \rightarrow \mathbb{R}$ be differentiable function on $I^{o}, a, b \in I$ with $a<b$. If $\left|f^{\prime}\right|^{q}$ is $h$-convex function, $0 \leq \lambda, \mu \leq 1$ and $n \in \mathbb{N}^{*}$, then for $q>1$, we have

$$
\left|\phi(n ; \lambda, \mu ; a, b)-\frac{1}{b-a}\left[\int_{a}^{\Omega_{1}} f(x) \mathrm{d} x+\int_{\Omega_{2}}^{b} f(x) \mathrm{d} x\right]\right| \leq \frac{b-a}{(n+1)^{2}}\left[J_{1}+J_{2}\right],
$$

where

$$
J_{1}=\left[\frac{1}{n+1} \int_{0}^{1}|1-\lambda-t|^{q}\left\{h(n+t)\left|f^{\prime}(a)\right|^{q}+h(1-t)\left|f^{\prime}(b)\right|^{q}\right\} \mathrm{d} t\right]^{\frac{1}{q}},
$$

and

$$
J_{2}=\left[\frac{1}{n+1} \int_{0}^{1}|\mu-t|^{q}\left\{h(t)\left|f^{\prime}(a)\right|^{q}+h(n+1-t)\left|f^{\prime}(b)\right|^{q}\right\} \mathrm{d} t\right]^{\frac{1}{q}} .
$$

Proof. By $h$-convexity of $\left|f^{\prime}(x)\right|^{q}$ on $[a, b]$, Lemma 1, and Hölder's integral inequality, we have

$$
\begin{aligned}
&\left|\phi(n ; \lambda, \mu ; a, b)-\frac{1}{b-a}\left[\int_{a}^{\Omega_{1}} f(x) \mathrm{d} x+\int_{\Omega_{2}}^{b} f(x) \mathrm{d} x\right]\right| \\
& \leq \frac{b-a}{(n+1)^{2}} \times\left[( \int _ { 0 } ^ { 1 } \mathrm { d } t ) ^ { 1 - \frac { 1 } { q } } \left\{\frac{1}{n+1} \int_{0}^{1}|1-\lambda-t|^{q}\right.\right. \\
&\left.\left(h(n+t)\left|f^{\prime}(a)\right|^{q}+h(1-t)\left|f^{\prime}(b)\right|^{q}\right) \mathrm{d} t\right\}^{\frac{1}{q}}+\left(\int_{0}^{1} \mathrm{~d} t\right)^{1-\frac{1}{q}} \\
&\left.\left\{\frac{1}{n+1} \int_{0}^{1}|\mu-t|^{q}\left(h(t)\left|f^{\prime}(a)\right|^{q}+h(n+1-t)\left|f^{\prime}(b)\right|^{q}\right) \mathrm{d} t\right\}^{\frac{1}{q}}\right] \\
&= \frac{b-a}{(n+1)^{2}}\left[J_{1}+J_{2}\right] .
\end{aligned}
$$

This completes the proof.

Now we will discuss special cases of Theorem 5.

(i) When $h(t)=1$

Corollary 16. Let $f: I \rightarrow \mathbb{R}$ be differentiable function on $I^{o}, a, b \in I$ with $a<b$. If $\left|f^{\prime}\right|^{q}$ is $P$-function, $0 \leq \lambda, \mu \leq 1$ and $n \in \mathbb{N}^{*}$, then we have

$\left|\phi(n ; \lambda, \mu ; a, b)-\frac{1}{b-a}\left[\int_{a}^{\Omega_{1}} f(x) \mathrm{d} x+\int_{\Omega_{2}}^{b} f(x) \mathrm{d} x\right]\right|$ 


$$
\leq \frac{(b-a)\left(\left|f^{\prime}(a)\right|^{q}+\left|f^{\prime}(b)\right|^{q}\right)^{\frac{1}{q}}}{(n+1)^{3}}\left[\left(\frac{(1-\lambda)^{q+1}+\lambda \lambda^{q+1}}{q+1}\right)^{\frac{1}{q}}+\left(\frac{(1-\mu)^{q+1}+\mu^{q+1}}{q+1}\right)^{\frac{1}{q}}\right] .
$$

(ii) When $h(t)=t$

Corollary 17. Let $f: I \rightarrow \mathbb{R}$ be differentiable function on $I^{o}, a, b \in I$ with $a<b$. If $\left|f^{\prime}\right|^{q}$ is classical convex function, $0 \leq \lambda, \mu \leq 1$ and $n \in \mathbb{N}^{*}$, then we have

$$
\begin{aligned}
& \left|\phi(n ; \lambda, \mu ; a, b)-\frac{1}{b-a}\left[\int_{a}^{\Omega_{1}} f(x) \mathrm{d} x+\int_{\Omega_{2}}^{b} f(x) \mathrm{d} x\right]\right| \\
& \leq \frac{b-a}{(n+1)^{3}}\left[\frac{1}{(q+1)(q+2)}\right]^{\frac{1}{q}} \times\left[\left\{| f ^ { \prime } ( a ) | ^ { q } \left((n q+2 n+1-\lambda)(1-\lambda)^{q+1}\right.\right.\right. \\
& \left.\left.\quad+((n+1) q+2(n+1)-\lambda) \lambda^{q+1}\right)+\left|f^{\prime}(b)\right|^{q}\left((q+1+\lambda)(1-\lambda)^{q+1}+\lambda^{q+2}\right)\right\}^{\frac{1}{q}} \\
& \quad+\left\{\left|f^{\prime}(a)\right|^{q}\left(\mu^{q+2}+(q+1+\mu)(1-\mu)^{q+1}\right)\right. \\
& \left.\left.\quad+\left|f^{\prime}(b)\right|^{q}\left(((n+1) q+2(n+1)-\mu) \mu^{q+1}+(n q+2 n+1-\mu)(1-\mu)^{q+1}\right)\right\}^{\frac{1}{q}}\right] .
\end{aligned}
$$

Letting $\lambda=\mu=\frac{1}{2}$ in Corollary 17 , we have

$$
\begin{aligned}
\phi\left(n ; \frac{1}{2}, \frac{1}{2} a, b\right)= & \frac{1}{n+1}\left[\frac{f(a)+f(b)}{2}+\frac{1}{2}\left\{f\left(\frac{n}{n+1} a+\frac{1}{n+1} b\right)\right.\right. \\
& \left.\left.+f\left(\frac{1}{n+1} a+\frac{n}{n+1} b\right)\right\}\right] .
\end{aligned}
$$

Corollary 18. Let $f: I \rightarrow \mathbb{R}$ be differentiable function on $I^{o}, a, b \in I$ with $a<b$. If $\left|f^{\prime}\right|^{q}$ is convex function, $q \geq 1, \lambda=\mu=\frac{1}{2}$ and $n \in \mathbb{N}^{*}$, then we have

$$
\begin{aligned}
& \left|\phi\left(n ; \frac{1}{2}, \frac{1}{2} a, b\right)-\frac{1}{b-a}\left[\int_{a}^{\Omega_{1}} f(x) \mathrm{d} x+\int_{\Omega_{2}}^{b} f(x) \mathrm{d} x\right]\right| \\
& \leq \frac{b-a}{2(n+1)^{3}}\left[\frac{1}{4(q+1)(q+2)}\right]^{\frac{1}{q}} \\
& \quad \times\left[\left\{\left|f^{\prime}(a)\right|^{q}((2 n q+4 n+1)+(2(n+1) q+4 n+3))+\left|f^{\prime}(b)\right|^{q}((2 q+3)+1)\right\}^{\frac{1}{q}}\right. \\
& \left.\quad+\left\{\left|f^{\prime}(a)\right|^{q}(1+(2 q+3))+\left|f^{\prime}(b)\right|^{q}((2(n+1) q+4 n+3)+(2 n q+4 n+1))\right\}^{\frac{1}{q}}\right] .
\end{aligned}
$$

Corollary 19. Let $f: I \rightarrow \mathbb{R}$ be differentiable function on $I^{o}, a, b \in I$ with $a<b$. If $\left|f^{\prime}\right|^{q}$ is convex function, $q \geq 1, \lambda=\mu=\frac{1}{2}$ and $n \in \mathbb{N}^{*}$ and

$$
\frac{f(a)+f(b)}{2}=f\left(\frac{a+b}{2}\right)
$$


then

$$
\begin{aligned}
& \left|\phi\left(n ; \frac{1}{2}, \frac{1}{2} a, b\right)-\frac{1}{b-a}\left[\int_{a}^{\Omega_{1}} f(x) \mathrm{d} x+\int_{\Omega_{2}}^{b} f(x) \mathrm{d} x\right]\right| \\
& \leq \frac{b-a}{2(n+1)^{3}}\left[\frac{1}{4(q+1)(q+2)}\right]^{\frac{1}{q}} \times\left\{\left[(2 n q+4 n+1)\left|f^{\prime}(a)\right|^{q}+(2 q+3)\left|f^{\prime}(b)\right|^{q}\right]^{\frac{1}{q}}\right. \\
& \quad+\left[(2(n+1) q+4 n+3)\left|f^{\prime}(a)\right|^{q}+\left|f^{\prime}(b)\right|^{q}\right]^{\frac{1}{q}} \\
& \quad+\left[\left|f^{\prime}(a)\right|^{q}+(2(n+1) q+4 n+3)\left|f^{\prime}(b)\right|^{q}\right]^{\frac{1}{q}} \\
& \left.\quad+\left[(2 q+3)\left|f^{\prime}(a)\right|^{q}+(2 n q+4 n+1)\left|f^{\prime}(b)\right|^{q}\right]^{\frac{1}{q}}\right\} .
\end{aligned}
$$

(iii) When $h(t)=t^{s}$

Corollary 20. Let $f: I \rightarrow \mathbb{R}$ be differentiable function on $I^{o}, a, b \in I$ with $a<b$. If $\left|f^{\prime}\right|^{q}$ is Breckner type of s-convex function, $0 \leq \lambda, \mu \leq 1$ and $n \in \mathbb{N}^{*}$, then we have

$$
\left|\phi(n ; \lambda, \mu ; a, b)-\frac{1}{b-a}\left[\int_{a}^{\Omega_{1}} f(x) \mathrm{d} x+\int_{\Omega_{2}}^{b} f(x) \mathrm{d} x\right]\right| \leq \frac{b-a}{(n+1)^{2}}\left[T_{1}+T_{2}\right] .
$$

where

$$
T_{1}=\left[\frac{1}{n+1} \int_{0}^{1}|1-\lambda-t|^{q}\left\{(n+t)^{s}\left|f^{\prime}(a)\right|^{q}+(1-t)^{s}\left|f^{\prime}(b)\right|^{q}\right\} \mathrm{d} t\right]^{\frac{1}{q}},
$$

and

$$
T_{2}=\left[\frac{1}{n+1} \int_{0}^{1}|\mu-t|^{q}\left\{t^{s}\left|f^{\prime}(a)\right|^{q}+(n+1-t)^{s}\left|f^{\prime}(b)\right|^{q}\right\} \mathrm{d} t\right]^{\frac{1}{q}} .
$$

(iv) When $h(t)=t^{-s}$

Corollary 21. Let $f: I \rightarrow \mathbb{R}$ be differentiable function on $I^{o}, a, b \in I$ with $a<b$. If $\left|f^{\prime}\right|^{q}$ is Godunova-Levin-Dragomir type of s-convex function, $0 \leq \lambda, \mu \leq 1$ and $n \in \mathbb{N}^{*}$, then we have

$$
\left|\phi(n ; \lambda, \mu ; a, b)-\frac{1}{b-a}\left[\int_{a}^{\Omega_{1}} f(x) \mathrm{d} x+\int_{\Omega_{2}}^{b} f(x) \mathrm{d} x\right]\right| \leq \frac{b-a}{(n+1)^{2}}\left[T_{1}^{*}+T_{2}^{*}\right],
$$

where

$$
T_{1}^{*}=\left[\frac{1}{n+1} \int_{0}^{1}|1-\lambda-t|^{q}\left\{(n+t)^{-s}\left|f^{\prime}(a)\right|^{q}+(1-t)^{-s}\left|f^{\prime}(b)\right|^{q}\right\} \mathrm{d} t\right]^{\frac{1}{q}},
$$

and

$$
T_{2}^{*}=\left[\int_{0}^{1}|\mu-t|^{q}\left\{t^{-s}\left|f^{\prime}(a)\right|^{q}+(n+1-t)^{-s}\left|f^{\prime}(b)\right|^{q}\right\} \mathrm{d} t\right]^{\frac{1}{q}} .
$$

(v) When $h(t)=t(1-t)$ 
Corollary 22. Let $f: I \rightarrow \mathbb{R}$ be differentiable function on $I^{o}, a, b \in I$ with $a<b$. If $\left|f^{\prime}\right|^{q}$ is tgs-convex function, $0 \leq \lambda, \mu \leq 1$ and $n \in \mathbb{N}^{*}$, then we have

$$
\begin{aligned}
& \left|\phi(n ; \lambda, \mu ; a, b)-\frac{1}{b-a}\left[\int_{a}^{\Omega_{1}} f(x) \mathrm{d} x+\int_{\Omega_{2}}^{b} f(x) \mathrm{d} x\right]\right| \\
& \leq \\
& \leq\left(\frac{b-a}{(n+1)^{3}}\right)\left(\frac{1}{(q+1)(q+2)(q+3)}\right)^{\frac{1}{q}} \\
& \quad\left[\left|f^{\prime}(a)\right|^{q} \times\left\{(1-\lambda)^{q+1}\left[n(1-n)(q+2)(q+3)+(1-2 n)(1-\lambda)(q+3)-2(1-\lambda)^{2}\right]\right.\right. \\
& \left.\quad-(-\lambda)^{q+1}\left[n(1+n)(q+2)(q+3)-(1+2 n)(q+3) \lambda+2 \lambda^{2}\right]\right\} \\
& \left.\quad+\left|f^{\prime}(b)\right|^{q} \times\left\{(1-\lambda)^{q+2}\left[q+3-2(1-\lambda)-2(1-\lambda)^{2}\right]-(-\lambda)^{q+2}[2 \lambda-(q+3)]\right\}\right]^{\frac{1}{q}} \\
& \quad+\left[\left|f^{\prime}(a)\right|^{q} \times\left\{\mu^{q+2}[(q+3)-2 \mu](q+1)-(\mu-1)^{q+2}[(q+3)+2(\mu-1)]\right\}\right. \\
& \quad+\left|f^{\prime}(b)\right|^{q} \times\left\{(\mu)^{q+1}\left[-n(1+n)(q+2)(q+3)+(1+2 n)(q+3) \mu-2 \mu^{2}\right]\right. \\
& \quad-(\mu-1)^{q+1}\left[n(n-1)(q+2)(q+3)(\mu-1)^{q+1}\right] \\
& \left.\left.\quad-(\mu-1)^{q+1}\left[(1-2 n)(q+3)(\mu-1)+2(\mu-1)^{2}\right]\right\}\right]^{\frac{1}{q}} .
\end{aligned}
$$

\section{Applications}

For $a>0, b>0$ and $0 \leq \omega<\infty$, define

$$
\begin{gathered}
A(a, b)=\frac{a+b}{2}, \quad G(a, b)=\sqrt{a b}, \quad H(a, b)=\frac{2 a b}{a+b} . \\
L(a, b)= \begin{cases}{\left[\frac{b^{s+1}-a^{s+1}}{(s+1)(b-a)}\right],} & a \neq b \\
a, & a=b,\end{cases} \\
I(a, b)= \begin{cases}\frac{1}{e}\left(\frac{b^{b}}{a^{a}}\right)^{\frac{1}{b-a}}, & a \neq b \\
a, & a=b,\end{cases} \\
H_{\omega, s}(a, b)= \begin{cases}{\left[\frac{a^{s}+(a b)^{s / 2} \omega+b^{s}}{\omega+2}\right],} & s \neq 0 \\
\sqrt{a b}, & s=0 .\end{cases}
\end{gathered}
$$

These means of two positive number $a$ and $b$ are, respectively, called the arithmetic, harmonic, generalized logarithmic, identric, and Heronion means respectively. Now applying Corollary 17 to $f(x)=x^{s}$ for $s \neq 0$ and $x>0$ leads to the following inequality for means. 
Theorem 6. Let $a, b>0, q \geq 1$, either $s>1$ and $(s-1) q \geq 1$ or $s<0$, then

$$
\begin{aligned}
\mid & \frac{A}{\left(a^{s}, b^{s}\right)+A\left(\left(\frac{1}{n+1} a+\frac{n}{n+1} b\right)^{s},\left(\frac{n}{n+1} a+\frac{1}{n+1} b\right)^{s}\right)} \\
& +\left(\frac{n-1}{n+1}\right) L^{s}\left(\frac{1}{n+1} a+\frac{n}{n+1} b, \frac{n}{n+1} a+\frac{1}{n+1} b\right)-L^{s}(a, b) \mid \\
\leq & \frac{b-a}{2(n+1)^{3}}|s|^{q}\left[\frac{1}{4(q+1)(q+2)}\right]^{\frac{1}{q}} \\
& \times\left\{\left[(2 n q+4 n+1) a^{(s-1) q}+(2 q+3) b^{(s-1) q}\right]^{\frac{1}{q}}\right. \\
& +\left[(2(n+1) q+4 n+3) a^{(s-1) q}+b^{(s-1) q}\right]^{\frac{1}{q}} \\
& +\left[a^{(s-1) q}+(2(n+1) q+4 n+3) b^{(s-1) q}\right]^{\frac{1}{q}} \\
& \left.+\left[(2 q+3) a^{(s-1) q}+(2 n q+4 n+1) b^{(s-1) q}\right]^{\frac{1}{q}}\right\} .
\end{aligned}
$$

Taking $f(x)=\ln x$ for $x>0$ in Corollary 17 results in the following inequalities for means.

Theorem 7. Let $a, b>0$ and $q \geq 1$, we get

$$
\begin{aligned}
& \frac{\ln G(a, b)+\ln G\left(\frac{1}{n+1} a+\frac{n}{n+1} b, \frac{n}{n+1} a+\frac{1}{n+1} b\right)}{n+1} \\
& +\frac{n-1}{n+1} \ln I\left(\frac{1}{n+1} a+\frac{n}{n+1} b, \frac{n}{n+1} a+\frac{1}{n+1} b\right)-\ln I(a, b) \\
& \leq \frac{b-a}{2(n+1)^{3}}|s|^{q}\left[\frac{1}{4(q+1)(q+2)}\right]^{\frac{1}{q}} \\
& \times\left\{\left[\frac{2 n q+4 n+1}{a^{q}}+\frac{2 q+3}{b^{q}}\right]^{\frac{1}{q}}+\left[\frac{2(n+1) q+4 n+3}{a^{q}}+\frac{1}{b^{q}}\right]^{\frac{1}{q}}\right. \\
& \left.+\left[\frac{1}{a^{q}}+\frac{2(n+1) q+4 n+3}{b^{q}}\right]^{\frac{1}{q}}+\left[\frac{2 q+3}{a^{q}}+\frac{2 n q+4 n+1}{b^{q}}\right]^{\frac{1}{q}}\right\} .
\end{aligned}
$$

Theorem 8. For $b>a>0, w \geq 0$, and $s \geq 4$ or $0 \neq s<1$, one has

$$
\mid \frac{1}{n+1}\left[\frac{H_{w, s}^{s}(a, b)}{H\left(a^{s}, b^{s}\right)}+\frac{1}{2}\left\{H_{w, s}^{s}\left(\frac{n}{n+1} \frac{a}{b}+\frac{1}{n+1} \frac{b}{a}, 1\right)\right.\right.
$$




$$
\begin{aligned}
& \left.\left.+H_{w, s}^{s}\left(\frac{1}{n+1} \frac{a}{b}+\frac{n}{n+1} \frac{b}{a}, 1\right)\right\}\right]-H_{w, s}^{s}\left(\frac{L\left(a^{2}, b^{2}\right)}{G^{2}(a, b)}\right) \\
& -\left(\frac{1-n}{1+n}\right) H_{w, s}^{s}\left(\frac{L\left(\frac{1}{n+1} a^{2}+\frac{n}{n+1} b^{2}, \frac{1}{n+1} a^{2}+\frac{1}{n+1} b^{2}\right)}{G^{2}(a, b)}\right) \mid \\
& \leq \frac{(b-a)|s|}{(n+1)^{2}}\left[\frac{A(a, b) A\left(a^{2(s-1)}, b^{2(s-1)}\right)}{(w+2) G^{2 s}(a, b)}+\frac{A(a, b) A\left(a^{s-2}, b^{s-2}\right)}{(w+2) G^{s}(a, b)}\right] .
\end{aligned}
$$

Proof. Let $f(x)=\frac{x^{s}+w x^{s / 2}+1}{w+2}$ for $s \notin(1,4)$ and $x>0$, then

$$
\begin{gathered}
f^{\prime}(x)=\frac{s}{w+2}\left(x^{s-1}+\frac{w}{2} x^{s / 2-1}\right) \\
\int f(x) \mathrm{d} x=\frac{1}{w+2}\left(\frac{x^{s+1}}{s+1}+\frac{w x^{s / 2+1}}{s / 2+1}+x\right) .
\end{gathered}
$$

Now

$$
\begin{aligned}
& \frac{1}{\frac{b}{a}-\frac{a}{b}}\left[\int_{\frac{a}{b}}^{\frac{n}{n+1} \frac{a}{b}+\frac{1}{n+1} \frac{b}{a}} f(x) \mathrm{d} x+\int_{\frac{1}{n+1} \frac{a}{b}+\frac{n}{n+1} \frac{b}{a}}^{\frac{b}{a}} f(x) \mathrm{d} x\right] \\
& =\frac{\left(\frac{n}{n+1} a^{2}+\frac{1}{n+1} b^{2}\right)^{s+1}-\left(\frac{n}{n+1} a^{2}+\frac{1}{n+1} b^{2}\right)^{s+1}}{(w+2)(s+1)\left(b^{2}-a^{2}\right)(a b)^{s}} \\
& \quad+w \frac{\left(\frac{n}{n+1} a^{2}+\frac{1}{n+1} b^{2}\right)^{\frac{s}{2}+1}-\left(\frac{n}{n+1} a^{2}+\frac{1}{n+1} b^{2}\right)^{\frac{s}{2}+1}}{(w+2)\left(\frac{s}{2}+1\right)\left(b^{2}-a^{2}\right)(a b)^{\frac{s}{2}}} \\
& \quad+\frac{1}{w+2}+\frac{\left(b^{2}\right)^{s+1}-\left(a^{2}\right)^{s+1}}{(w+2)(s+1)\left(b^{2}-a^{2}\right)(a b)^{s}} \\
& \left.\quad+w \frac{\left(b^{2}\right)^{\frac{s}{2}}-\left(a^{2}\right)^{\frac{s}{2}}}{(w+2)\left(\frac{s}{2}+1\right)\left(b^{2}-a^{2}\right)(a b)^{\frac{s}{2}}+\frac{1}{w+2}}\right)+H_{w, s}^{s}\left(\frac{L\left(a^{2}, b^{2}\right)}{G^{2}(a, b)}\right), \\
& =\left(\frac{1-n}{1+n}\right) H_{w, s}^{s}\left(\frac{L\left(\frac{1}{n+1} a^{2}+\frac{n}{n+1} b^{2}, \frac{1}{n+1} a^{2}+\frac{1}{n+1} b^{2}\right)}{G^{2}(a, b)}\right)
\end{aligned}
$$

and

$$
\begin{aligned}
& \phi\left(n ; \frac{1}{2}, \frac{1}{2}, a, b\right) \\
& =\frac{1}{n+1}\left[\frac{f(a)+f(b)}{2}+\frac{1}{2}\left\{f\left(\frac{n}{n+1} a+\frac{1}{n+1} b\right)+f\left(\frac{1}{n+1} a+\frac{n}{n+1} b\right)\right\}\right] \\
& =\frac{1}{n+1}\left[\frac{\left(\frac{a}{b}\right)^{s}+w\left(\frac{a}{b}\right)^{s / 2}+1}{2(w+2)}+\frac{\left(\frac{b}{a}\right)^{s}+w\left(\frac{b}{a}\right)^{s / 2}+1}{2(w+2)}\right.
\end{aligned}
$$




$$
\begin{aligned}
& +\frac{1}{2(w+2)}\left\{\left(\frac{n}{n+1} \frac{a}{b}+\frac{1}{n+1} \frac{b}{a}\right)^{s}+w\left(\frac{n}{n+1} \frac{a}{b}+\frac{1}{n+1} \frac{b}{a}\right)^{s / 2}+1\right. \\
& \left.\left.+\left(\frac{1}{n+1} \frac{a}{b}+\frac{n}{n+1} \frac{b}{a}\right)^{s}+w\left(\frac{1}{n+1} \frac{a}{b}+\frac{n}{n+1} \frac{b}{a}\right)^{s / 2}+1\right\}\right] \\
& =\frac{1}{n+1}\left[\frac{H_{w, s}^{s}(a, b)}{H\left(a^{s}, b^{s}\right)}+\frac{1}{2}\left\{H_{w, s}^{s}\left(\frac{n}{n+1} \frac{a}{b}+\frac{1}{n+1} \frac{b}{a}, 1\right)\right.\right. \\
& \left.+H_{w, s}^{s}\left(\frac{1}{n+1} \frac{a}{b}+\frac{n}{n+1} \frac{b}{a}, 1\right)\right\} .
\end{aligned}
$$

In virtue of Corollary 19, it follows that

$$
\begin{aligned}
\left|\phi(n ; \lambda, \mu, a, b)-\frac{1}{b-a}\left[\int_{a}^{\Omega_{1}} f(x) \mathrm{d} x+\int_{\Omega_{2}}^{b} f(x) \mathrm{d} x\right]\right| \\
=\mid \frac{1}{n+1}\left[\frac{H_{w, s}^{s}(a, b)}{H\left(a^{s}, b^{s}\right)}+\frac{1}{2}\left\{H_{w, s}^{s}\left(\frac{n}{n+1} \frac{a}{b}+\frac{1}{n+1} \frac{b}{a}, 1\right)\right.\right. \\
\left.\left.\quad+H_{w, s}^{s}\left(\frac{1}{n+1} \frac{a}{b}+\frac{n}{n+1} \frac{b}{a}, 1\right)\right\}\right]-H_{w, s}^{s}\left(\frac{L\left(a^{2}, b^{2}\right)}{G^{2}(a, b)}\right) \\
\quad-\left(\frac{1-n}{1+n}\right) H_{w, s}^{s}\left(\frac{L\left(\frac{1}{n+1} a^{2}+\frac{n}{n+1} b^{2}, \frac{1}{n+1} a^{2}+\frac{1}{n+1} b^{2}\right)}{G^{2}(a, b)}\right) \mid .
\end{aligned}
$$

On the other hand, we have

$$
\begin{aligned}
& \frac{\frac{b}{a}-\frac{a}{b}}{4(n+1)^{2}}\left[\left|f^{\prime}\left(\frac{a}{b}\right)\right|+\left|f^{\prime}\left(\frac{b}{a}\right)\right|\right] \\
& =\frac{b^{2}-a^{2}}{4(n+1)^{2} a b}\left(\frac{|s|}{w+2}\right)\left\{\left[\left(\frac{b}{a}\right)^{s-1}+\left(\frac{a}{b}\right)^{s-1}\right]+\frac{w}{2}\left[\left(\frac{b}{a}\right)^{s / 2-1}+\left(\frac{a}{b}\right)^{s / 2-1}\right]\right\} \\
& =\frac{(b-a)|s|}{(n+1)^{2}}\left[\frac{A(a, b) A\left(a^{2(s-1)}, b^{2(s-1)}\right)}{(w+2) G^{2 s}(a, b)}+\frac{A(a, b) A\left(a^{s-2}, b^{s-2}\right)}{(w+2) G^{s}(a, b)}\right] .
\end{aligned}
$$

This completes the proof.

\section{CONCLUSION}

In this article, we have derived a new auxiliary result. With the help of this result we obtained several new Hermite-Hadamard type of integral inequalities. Numerous new special cases which can be deduced from our main results are also discussed in detail. At the last we have discussed applications of our results to means of real numbers. It is expected that the ideas and techniques of this paper may stimulate further research in the field. 


\section{REFERENCES}

[1] W. W. Breckner, "Stetigkeitsaussagen für eine klasse verallgemeinerter konvexer funktionen in topologischen linearen räumen," Publ. Inst. Math., no. 23, pp. 13-20, 1978.

[2] G. Cristescu and L. Lupsa, Non-connected Convexities and Applications. Kluwer Academic Publishers, Dordrecht, Holland, 2002.

[3] G. Cristescu, M. A. Noor, and M. U. Awan, "Bounds of the second degree cumulative frontier gaps of functions with generalized convexity," Carpath. J. Math., vol. 31, no. 2, pp. 173-180, 2015.

[4] S. S. Dragomir, "Inequalities of Jensen type for $h$-convex functions on linear spaces," Mathmat. Moravica, vol. 19, no. 1, pp. 107-121, 2015, doi: http://dx.doi.org/10.5937/MatMor1501107S.

[5] S. S. Dragomir and R. P. Agarwal, "Two inequalities for differentiable mappings and applications to special means of real numbers and to trapezoidal formula," Appl. Math. Lett., vol. 11, no. 5, pp. 91-95, 1998.

[6] S. S. Dragomir and C. E. M. Pearce, Selected Topics on Hermite-Hadamard Inequalities and Applications. Victoria University Australia, 2000.

[7] S. S. Dragomir, J. Pecaric, and L. E. Pearson, "Some inequalities of Hadamard type," Soochow J. Math., vol. 21, no. 3, pp. 335-341, 1995.

[8] T. S. Du, Y. J. Li, and Z. Q. Yang, “A generalization of Simpson's inequality via differentiable mapping using extended (s,m)-convex functions," Appl. Math. Comput., vol. 293, pp. 358-369, 2017, doi: http://dx.doi.org/10.1016/j.amc.2016.08.045.

[9] T. S. Du, J. Liao, and Y. J. Li, "Properties and integral inequalities of Hadamard-Simpson type for the generalized $(s, m)$-preinvex functions," J. Nonlinear Sci. Appl., vol. 9, no. 5, pp. 3112-3126, 2016, doi: http://dx.doi.org/10.22436/jnsa.009.05.102.

[10] E. K. Godunova and V. Levin, "Neravenstva dlja funkcii sirokogo klassa, soderzascego vypuklye, monotonnye inekotorye drugie vidy funkcii," Vycislitel, 1985.

[11] C. P. Niculescu and L. E. Person, "Convex functions and their applications, a contemporary approach," vol. 23, 2006.

[12] M. A. Noor, F. Qi, and M. U. Awan, "Some Hermite-Hadamard type inequalities for $\log -h$-convex functions," Analysis, vol. 33, no. 4, pp. 1-9, 2013, doi: http://dx.doi.org/10.1524/anly.2013.1175.

[13] M. Tunc, E. Gov, and U. Sanal, "On $\operatorname{tgs}$-convex function and their inequalities," Facta universitatis (NIS) Series: Math. Infor., vol. 30, no. 5, pp. 679-691, 2015.

[14] S. Varosanec, "On h-convexity," J. Math. Anal. Appl., vol. 326, no. 1, pp. 303-311, 2007, doi: https://doi.org/10.1016/j.jmaa.2006.02.086.

[15] B. Xi and F. Qi, "Some integral inequalities of Hermite-Hadamard type for convex functions with applications to means," J. Func. Spaces, vol. 2012, pp. 1-14, 2012, doi: http://dx.doi.org/10.1155/2012/980438.

Authors' addresses

M. U. Awan

Department of Mathematics, GC University, Faisalabad, Pakistan

E-mail address: awan.uzairegmail.com

M. A. Noor

Department of Mathematics, COMSATS University Islamabad, Islamabad, Pakistan

E-mail address: noormas lamegmail.com 


\section{F. Safdar}

Department of Mathematics, COMSATS University Islamabad, Islamabad, Pakistan

E-mail address: farhat_900@yahoo.com

\section{A. Islam}

Department of Mathematics, GC University, Faisalabad, Pakistan

E-mail address: asmariaislamegmail.com

M. V. Mihai

Department Scientific-Methodical Sessions, Romanian Mathematical Society-branch Bucharest, Academy Street no. 14, RO-010014, Bucharest, Romania

E-mail address: marcelamihai58@yahoo.com

\section{K. I. Noor}

Department of Mathematics, COMSATS University Islamabad, Islamabad, Pakistan

E-mail address: khalidan@gmail.com 